\title{
Structural and Electronic Properties of Clean and Defected Si-SiC(001) Surfaces
}

\author{
G Galli \\ F. Gygi \\ A Catellani \\ This paper was prepared for submittal to the \\ 10th Conference on Semiconducting and Insulating Materials \\ Berkeley, $C A$ \\ June 1-5, 1998
}

June 1998

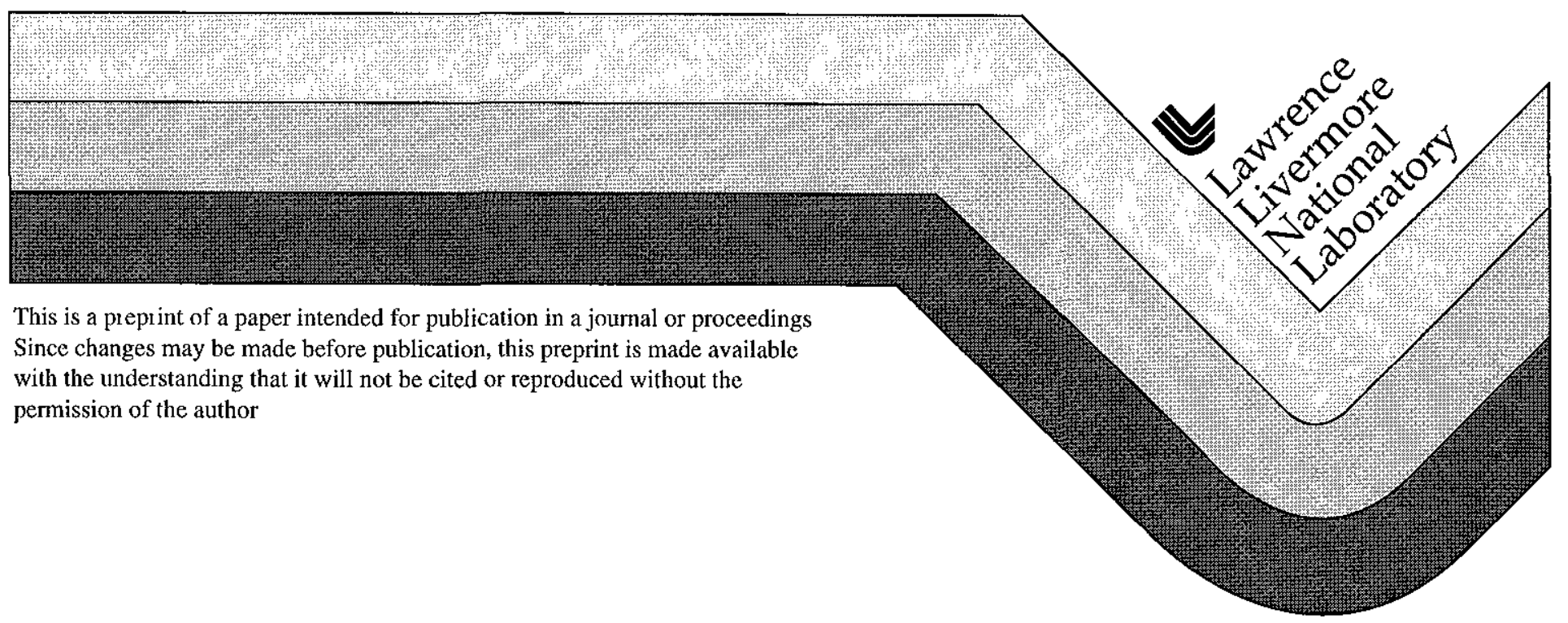




\section{DISCLAIMER}

This document was prepared as an account of work sponsored by an agency of the United States Government Neither the United States Government nor the University of California nor any of their employees, makes any warranty, express or implied, or assumes any legal liability or responsibility for the accuracy, completeness, or usefulness of any information, apparatus, product, or process disclosed, or represents that its use would not infringe privately owned rights Reference herein to any specific commercial product, process, or service by trade name, trademark, manufacturer, or otherwise, does not necessarily constitute or imply its endorsement, recommendation, or favoring by the United States Government or the University of California The views and opinions of authors expressed herein do not necessarily state or reflect those of the United States Government or the University of California, and shall not be used for advet tising or product endorsement purposes 


\title{
Structural and electronic properties of clean and defected $\mathrm{Si}-\mathrm{SiC}(001)$ surfaces
}

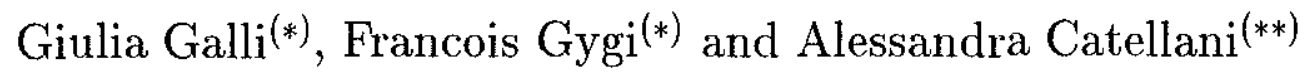 \\ (*) Lawrence Livermore National Laboratory, P.O.Box 808, \\ Livermore, CA 94551, USA. \\ (**) CNR-MASPEC, Via Chiavari, 18/A, I-43100 Parma, Italy.
}

\begin{abstract}
We have studied the reconstructions and electronic properties of both clean and defected Si-terminated (001) surfaces of cubic SiC, by performing first principles computations within density functional theory. We find that the unstrained bulk exhibits a stable $\mathrm{p}(2 \times 1)$ reconstruction, whereas a bulk under tensile stiess shows a $\mathrm{c}(4 \times 2)$ reconstruction Furthermore our calculations indicate that ad-dimers are common defects on the Si-terminated $\mathrm{SiC}(001)$ surface These results permit the interpretation of recent STM and X-1ay-photoemission experimental data
\end{abstract}

\section{A Introduction}

The characterization of $\mathrm{SiC}$ suffaces is an essential prerequisite to understanding the growth of Silicon Carbide, as well as its applications as a semiconductor for high-power, high-temperature and high-1adiation environments [1].

Here we focus on the Si-terminated (001) surface of the cubic polytype of $\mathrm{SiC}(\beta-\mathrm{SiC})$, which is one of the most studied surfaces, although not yet well characterized [2] There are two main issues which need to be addressed in order to understand the physical properties of $\mathrm{SiC}$ surfaces (i) the influence of stress on the surface reconstr uction and (ii) the modifications introduced by defects on the surface structure and electronic properties Stress effects on cubic $\mathrm{SiC}$ surfaces represent a key issue since cubic SiC films are presently prepared by chemical vapor deposition on $\mathrm{Si}(001)$ substrates The lattice mismatch between $\mathrm{Si}$ and $\mathrm{SiC}$ is almost $20 \%$, and thus $\mathrm{SiC}$ samples grown on $\mathrm{Si}$ are expected to be strained Furthemore stoichiometric Si-terminated $\mathrm{SiC}(001)$ surfaces are prepared by evaporating excess-Si, and the presence of defects such as missing- and ad-dimers and missing- and ad-atoms is expected on the surface The presence of defects on $\mathrm{Si-SiC}(001)$ is clearly visible from STM images [3] although the type of defects has not yet been identified 
In this paper we report about theoretical studies of the influence of stress (section $\mathrm{B}$ ) and defects (section $\mathrm{C}$ ) on the properties of $\mathrm{Si}-\mathrm{SiC}(001)$ Our investigations have been carried out by performing a series of ab-initio molecular dynamics calculations within the local density functional approximation, using pseudopotentials and plane wave basis sets $[4,5]$

\section{B Clean Si-terminated surface}

Experimentally both $\mathrm{p}(2 \times 1)[2]$ and $c(4 \times 2)[2,3]$ patterns have been observed on clean $\mathrm{Si}-\mathrm{SiC}(001)$, with $\mathrm{p}(2 \times 1)$ reconstructions being often seen in areas of missing dimers [3] and low coverage The results of our calculations show that an unstrained bulk exhibits a $\mathrm{p}(2 \mathrm{x} 1)$ reconstruction, which is under tensile stress In agreement with Sabisch et al [6], we have found that the $\mathrm{p}(2 \times 1)$ reconstruction is characterized by dimer 1ows, with dimess much longer $(2.6 \AA)$ than those of $\mathrm{Si}(001)(\simeq 23 \AA)$ These dimers are weakly bonded, with no important hybridization involved. The presence of weak bonds on the surface has not been confirmed experimentally, and the only fit to LEED data available in the literature points at shorter dimers [7] However very recent ARUPS data [8] are consistent with models implying a weak bonding of the $\mathrm{Si}$ dimers. In our calculations we found that the $\mathrm{p}(2 \times 1)$ reconstructed surface is non metallic at least up to $400 \mathrm{~K}$ The surface has a gap between $\pi^{*}$-like antibonding states and $\sigma$ bonding states This is different from the electronic structure of the $\mathrm{Si}(001)$ and C(001) surfaces where the reconstruction opens a band gap between $\pi$ and $\pi^{*}$ surface states.

When applying small stresses to the cubic SiC bulk, we have obser ved a symmetry breaking of the surface reconstruction, leading to a $c(4 \times 2)$ patten [4]. In our calculation, the $c(4 \times 2)$ surface geometry is characterized by alternating unbuckled short and long dimers, the short dimers having a component perpendicular to the surface smaller than the long ones The dimer bond lengths are 254 and $262 \AA$ in the case, eg, of a $3 \%$ strained bulk This surface geometry is in agreement with the alternating-up-and-down-dimer (AUDD) model proposed on the basis of STM experiments [3] Similarly to the $\mathrm{p}(2 \times 1)$ reconstruction, the $c(4 \times 2)$ geometry exhibits a gap between antibonding occupied and bonding empty surface states

Calculated STM images for both $\mathrm{p}(2 \times 1)$ and $c(4 \times 2)$ reconstructed surfaces are displayed in Fig 1. The top panels display the derivative of the tunneling current with respect to applied voltage these images clearly show the $\pi$-like bonding states on the dimers. Bright spots appear on all dimess of the $\mathrm{p}(2 \times 1)$ dimer rows (left), on the contrary only the up dimers are visible on the $c(4 \times 2)$ rows (right). Constant curient plots at $V=-15 \mathrm{eV}$ (lower panels of Fig 1) show instead surface states having large components between dimers These are bonding and antibonding $\pi$-like states. On the $\mathrm{p}(2 \times 1)$ surface bright spots are identical on all dimers, while on the $\mathrm{c}(4 \times 2)$ surface they clearly show the difference in height between up and down dimess When lowering the voltage from -15 to $-3 \mathrm{eV}$ in constant current plots, we found spots localized also on dimers, showing the difference in height between up and down dimers for the $c(4 \times 2)$ reconstruction. As expected, lowering the voltage makes the bonding $\pi$ states have a larger contribution to the tunneling current. The image at $V=-3$ $\mathrm{eV}$ is in satisfactory agreement with measured [3] images at constant curlent, at the same voltage However the measured images show larger components on dimers, indicating that in 


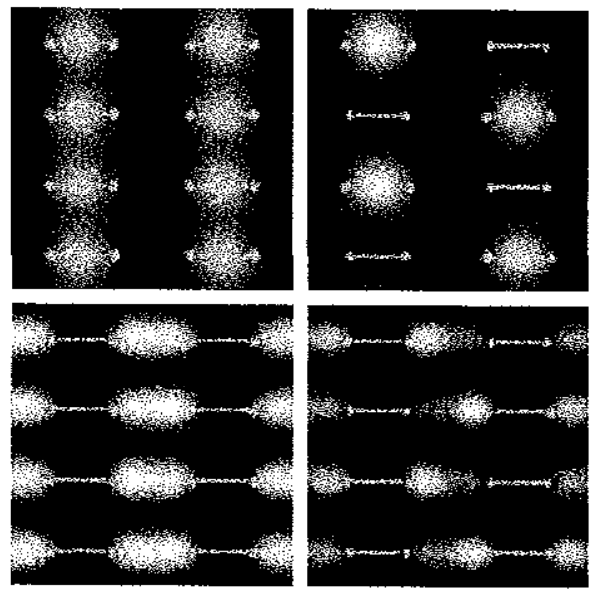

Figure 1 Computed STM images of the $\mathrm{p}(2 \mathrm{x} 1)$ (left panel) and $\mathrm{c}(4 \mathrm{x} 2)$ (right panel) reconstructions of $\mathrm{Si}-\mathrm{SiC}(001)$. the upper and lower panels show a plot of $\frac{\partial I\left(x, y, z_{0} ; V\right)}{\partial V} \propto$ $\Sigma_{i}\left|\psi_{i}\left(x, y, z_{0}\right)\right|^{2} f^{\prime}\left(\epsilon_{i}+e V\right)$ (see text) and $I(x, y, z, V)=I_{0}$, espectively, in both cases $\mathrm{V}=-1.5$ $\mathrm{eV}$. Here $I(x, y, z ; V)$ is the tunneling current computed within the Tersoff-Hamman approximation [10] at a given applied voltage $V$, the plot $I=I_{0}$ is drawn by considering all $z$ values between the surface and the top of the slab such that $I=I_{0}$

. From Ref [4]

our calculation of $I(x, y, z, V)$, antibonding components of surface states are weighted more than experimentally This difference between theory and experiment may be related to the tip induced electric field, which could change the energy separation between $\pi$ and $\pi^{*}$ surface states

\section{B Defected Si-terminated surfaces}

In order to make contacts with several experimental results indicating the presence of defects on Si-terminated surfaces [2], we have studied the structural modifications induced by point defects on the surface structure.

Since $\mathrm{p}(2 \mathrm{x} 1)$ reconstructions of $\mathrm{Si-} \mathrm{SiC}(001)$ have been often seen in areas of missing dimers [3], we first investigated a $\mathrm{p}(2 \times 1)$ reconstructed surface with missing dimers. We found that the removal of a dimer relieves surface stress and induces the formation of stronger bonds in four dimers surrounding the missing unit [4]. Nevertheless a dimer removal does not constitute a long ranged perturbation on the $\mathrm{p}(2 \times 1)$ reconstruction, whose symmetry and dimer bond lengths are basically unchanged The computed surface core level shifts (SCLS) [5] of atoms close to the missing dimer are similar to those of surface atoms on clean substrates $(09 \mathrm{eV}$, in our calculation), and vary between 08 and $1.1 \mathrm{eV}$.

We then considered an ad-atom and optimized two different surface geometries, with an extra atom between and on top of dimer lows, respectively The configuration of minimum energy cors esponds to the adatom sitting between rows, forming four long $(2.50 \AA)$, equivalent 
backbonds with the surface atoms The SCLS of the adatom - computed in the configuration of minimum energy - is very low $(\simeq 02 \mathrm{eV})$, compared to that of surface atoms

Finally we considered ad-dimess on a $\mathrm{p}(2 \mathrm{x} 1)$ terminated surface, and were inspired by recent investigations [9] of surfaces with excess $\mathrm{Si}$ atoms in order to determine a stable addimer geometry We have considered an ad-dimer between rows and optimized the total energy for geometries parallel and perpendicular to the $\mathrm{p}(2 \times 1)$ dimer rows. We have found that the perpendicular ad-dimer has a total energy about $06 \mathrm{eV}$ lower than the parallel one, and we have computed the SCLS for the configuration of minimum energy The chemical shifts of atoms belonging to an ad-dimer are larger than those of surface atoms, $\mathrm{i}$ e $\simeq 14$ $\mathrm{eV}$ higher in energy than the bulk value We note that the ad-dimer bond length is much

smaller than those of surface dimers, $228 \AA$, and thus the chemical bond of the ad-dimer is expected to be rather different from that of the weak, much longer surface dimeıs

Si-2p spectra [10] obtained in X-ray photoemission experiments show the presence of two peaks, a main peak (S) and a much less intense feature (S') centered at 0 5-0 $7 \mathrm{eV}$ and 1 2-1 7 $\mathrm{eV}$ above the bulk contribution, respectively In view of the results discussed above for SCLS, we have suggested that while surface atoms are responsible for the main peak $(S)$ observed experimentally in X-1ay photoemission spectra, ad-dimers are responsible for the less intense S' feature Our results [5] point at ad-dimers as common defects on $\mathrm{Si}-\mathrm{SiC}(001)$ surfaces

This work was supported by the U S Department of Energy under Contract No W-7405ENG-48.

[1] See, e.g., MRS bulletin 22, (1997)

[2] For a review see V. Bermudez, Phys. Stat. Sol A 201, 1997.

[3] P Soukiassian et al, Phys Rev Lett. 78, 907 (1997)

[4] A Catellani, G Galli, F Gygi and F. Pellacini, Phys. Rev B (May 1998)

[5] A Catellani, G Galli, F Gygi, Appl. Phys Lett (April 1998).

[6] M Sabisch et al Phys. Rev. B 53, 13121 (1996)

[7] J Powers et al Phys Rev B 44, 11159 (1991)

[8] P. Käckell et al Surf. Sci 391, L1183 (1997)

[9] F. Semond et al Phys. Rev Lett. 77, 2013 (1996); P Soukiassian et al Phys Rev Lett 79, 2498 (1997)

[10] V Bermudez and J Long, Appl Phys Lett 66, 475 (1995)

[11] J Tersoff and D Hamann, Phys. Rev B 31, 805 (1985) 


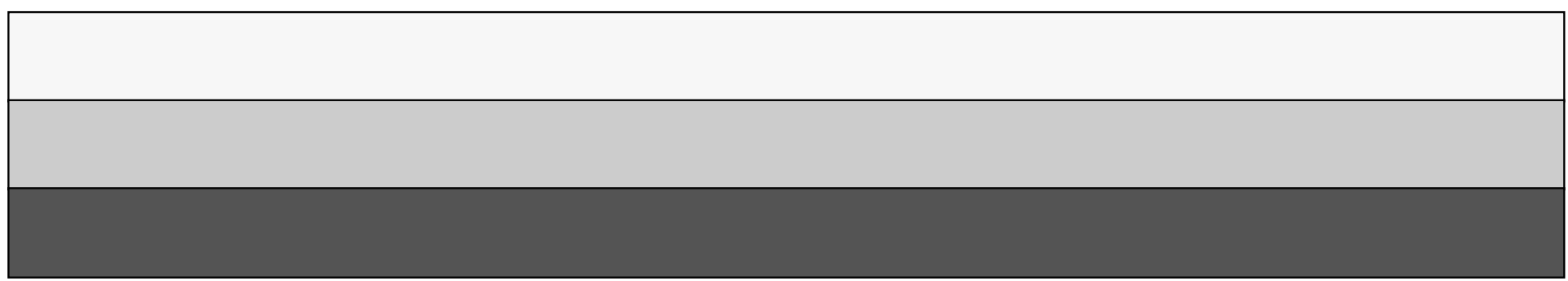

